\title{
Whole genome amplification of sodium bisulfite-treated DNA allows the accurate estimate of methylated cytosine density in limited DNA resources
}

\author{
Jonathan Mill1,2, Simin Yazdanpanah¹, Eva Gückel'1, Sigrid Ziegler¹, Zachary \\ Kaminsky ${ }^{1}$, and Arturas Petronis ${ }^{1}$ \\ ${ }^{1}$ Centre for Addiction and Mental Health, Toronto and ${ }^{2}$ Toronto Western Research \\ Institute, Toronto, ON, Canada
}

BioTechniques 41:603-607 (November 2006)

doi $10.2144 / 000112266$

Sodium bisulfite modification-based fine mapping of methylated cytosines represents the gold standard technique for DNA methylation studies. A major problem with this approach, however, is that it results in considerable DNA degradation, and large quantities of genomic DNA material are needed if numerous genomic regions are to be profiled. In this study, we examined whether whole genome amplification (WGA) techniques can be applied to sodium bisulfite-treated DNA and whether WGA would bias DNA methylation results. Sodium bisulfite-treated DNA was amplified using a standard WGA method: optimized primer-extension preamplification (PEP) with degenerate primers. Following the PCR of bisulfite-treated $D N A$, the DNA methylation profiles of specific DNA fragments were assessed using three approaches: (i) direct sequencing of the overall product; (ii) the sequencing of cloned PCR products; and (iii) methylation-sensitive single nucleotide primer extension (MS-SNuPE) and compared with those obtained from bisulfite-treated DNA not subjected to WGA. Our data indicates that the DNA methylation profiles obtained from WGA of sodium bisulfitetreated DNA are consistent with those obtained from non-WGA DNA. The average difference in methylation percentage calculated from the two sets of template using MS-SNuPE was 4\%. If our results are replicated on other genomic loci, WGA may become a useful technique in DNA methylation studies.

\section{INTRODUCTION}

Epigenetics refers to the heritable, but reversible, regulation of various genomic functions mediated through partially stable modifications of DNA and chromatin histones (1). DNA methylation [i.e., cytosine methylation (metC) at $\mathrm{CpG}$ and occasionally nonCpG sites] is one of the best-understood epigenetic mechanisms and has been investigated using a myriad of laboratory techniques (2). Over the last 15 years, the gold standard technique for fine mapping of ${ }^{\mathrm{met}} \mathrm{C}$ has been based on the treatment of genomic DNA with sodium bisulfite, which converts unmethylated cytosines to uracils (and subsequently, via PCR, to thymidines), while methylated cytosines are resistant to bisulfite and remain unchanged (3). After sodium bisulfite treatment, DNA regions of interest are amplified and sequenced to identify $\mathrm{C} \rightarrow \mathrm{T}$ transitions or stable $\mathrm{C}$ positions, respectively corresponding to unmethylated and methylated cytosines in the native DNA. Typically, PCR products are either sequenced directly to provide a strand-specific average sequence for the population of DNA molecules or cloned and sequenced to provide methylation maps of single DNA molecules $(3,4)$. An alternative approach to quantitatively assess the methylation level at specific cytosine sites within a product is methylationsensitive single nucleotide primer extension (Ms-SNuPE) $(5,6)$.

Conventional sodium bisulfite treatment is beset by a number of problems that result from the fact that to ensure the full conversion of unmethylated cytosines, the reaction is necessarily harsh and thus causes the large-scale degradation of genomic DNA (7). Alternative bisulfite-conversion protocols have been developed in which DNA is embedded in agarose during treatment to reduce DNA loss and ensure efficient conversion (8), but these procedures only partially reduce the degradation and are not suited to automated analysis. In many epigenetic studies, the amount of genomic DNA starting material is limited, especially in experiments utilizing valuable clinical samples, for example oocytes, laser capture microdissected cells, and microscope slides. Following bisulfite treatment, converted DNA is single-stranded and prone to further denaturation unless stored at $-80^{\circ} \mathrm{C}$. The degradation of DNA during and after sodium bisulfite treatment is a major hurdle to successful studies of DNA methylation.

In this report, we examine the applicability of whole genome amplification (WGA) to bisulfite-treated DNA. WGA methods are routinely used on normal genomic DNA for genotyping and sequence analysis when the amount of starting template is extremely low. One common application of WGA, for example, is in forensic analyses where it is used to improve both the quality and quantity of DNA and allows accurate genetic profiling from single cells (9). Two commonly used WGA strategies are $(i)$ primer extension preamplification (PEP), a Taq DNA polymerase PCR-based reaction (10) and (ii) multiple displacement amplification (MDA), an isothermal genome amplification using $\phi 29$ DNA polymerase (11). Both methods are utilized widely for genotyping, with several studies demonstrating the reliability of data produced from WGA templates $(12,13)$. In this study, we have primarily used an optimized version of the original PEP protocol to amplify sodium bisulfitetreated DNA. In addition, we tested a commercially available multiple displacement amplification (MDA) protocol (REPLI-g ${ }^{\circledR}$ ) on a number of samples. Following gene-specific PCR, we assessed methylation profiles using three approaches: $(i)$ direct sequencing of the entire product; $(i i)$ the sequencing of cloned PCR products; and (iii) single-nucleotide primer-extension, 
and compared data obtained from usual bisulfite DNA (non-WGA DNA) to that obtained from WGA bisulfite-treated DNA (WGA DNA).

\section{MATERIALS AND METHODS}

\section{Bisulfite Treatment of Genomic DNA}

Bisulfite treatment was performed using a standard protocol as described by Clark et al. (14). Briefly, approximately $500 \mathrm{ng}$ genomic DNA was denatured in $0.3 \mathrm{M} \mathrm{NaOH}$ for $15 \mathrm{~min}$ at $37^{\circ} \mathrm{C}$. After adding freshly prepared $3.5 \mathrm{M}$ sodium metabisulfite and 1 $\mathrm{mM}$ hydroquinone solution (both from Sigma, St. Louis, MO, USA), samples were subjected to a 5-h incubation at $55^{\circ} \mathrm{C}$ under exclusion of light. The samples were then purified using MinElute ${ }^{\circledR}$ PCR purification columns (Qiagen, Valencia, CA, USA). Recovered samples were desulfonated in $0.3 \mathrm{M} \mathrm{NaOH}$ for $15 \mathrm{~min}$ at $37^{\circ} \mathrm{C}$ and neutralized. DNA was precipitated overnight in ethanol at $-20^{\circ} \mathrm{C}$ and resuspended in $50 \mu \mathrm{L}$ elution buffer (Buffer EB; Qiagen).

\section{Whole Genome Amplification with PEP and MDA}

Two WGA methods were utilized. First, we performed a modified version of the original PEP protocol described by Zhang et al. (10). Briefly, $2 \mu \mathrm{L}$ bisulfite-treated DNA, corresponding to approximately $20 \mathrm{ng}$ of the original DNA template, were amplified in a $50-\mu \mathrm{L}$ reaction containing 200 pmol 15-mer degenerate primers $\left(5^{\prime}\right.$ NNNNNNNNNNNNNNN-3') and 0.4 $\mathrm{mM}$ dNTPs and cycled using standard WGA conditions (see Reference 10). Fifty primer-extension cycles were performed, with each cycle consisting of a 1-min denaturing step at $95^{\circ} \mathrm{C}$, a 2 -min annealing step at $37^{\circ} \mathrm{C}$, a programmed ramping step of $10 \mathrm{~s} /{ }^{\circ} \mathrm{C}$, and a 4-min incubation at $55^{\circ} \mathrm{C}$. Each reaction was done in triplicate (or quadruplicate), and the final products pooled giving a total of 150-200 $\mu \mathrm{L}$ PEP product resulting from approximately $60-80 \mathrm{ng}$ of the original DNA template. Following PEP, excess degenerate primers and deoxy-

A

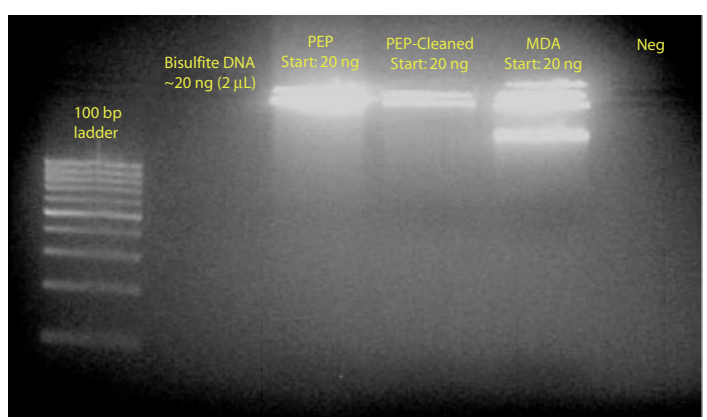

B

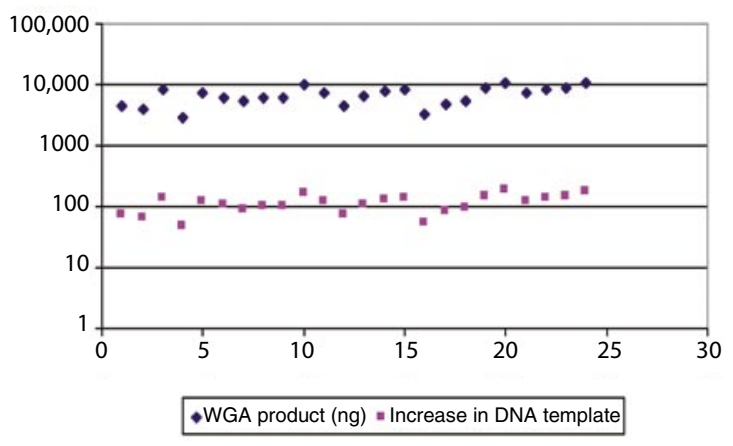

Figure 1. Whole genome amplification (WGA) results in a large increase in bisulfitetreated DNA template. (A) Representative WGA products obtained from standard sodium bisulfite-treated DNA starting material. Products in lanes 3 and 4 [primer-extension preamplification (PEP)] and lane 5 [multiple displacement amplification (MDA)] result from approximately $20 \mathrm{ng}$ original DNA [ $2 \mu \mathrm{L}$ sodium bisulfite treatment reaction (lane 2)]. Standard, non-WGA bisulfitetreated DNA is not visible, but both WGA methods produce clearly visible DNA smears of high genomic weight, demonstrating efficient amplification. Marker is the GeneRuler ${ }^{\mathrm{TM}}$ 100-bp DNA ladder (Fermentas, Hanover, MD, USA). Results from spectrophotometric analysis of 25 representative PEP-amplified products. The average yield from the pooled PEP replicates was $6.8 \mu \mathrm{g}( \pm 2.2$ $\mu \mathrm{g})$. Assuming no degradation during the sodium bisulfite conversion reaction, this represents a 113-fold $( \pm 37)$ increase from the initial starting genomic DNA (60 ng).

nucleotides were optionally removed using MinElute PCR purification columns, and products were eluted in $50 \mu \mathrm{L}$ Buffer EB. Second, MDA was performed on some samples using the REPLI-g kit (Qiagen) as described in the manufacturer's protocol. Following WGA, cleaned products were quantified spectrophotometrically and stored at $4^{\circ} \mathrm{C}$ or transferred to $-20^{\circ} \mathrm{C}$ for longterm storage.

\section{Methylation Profiling}

Several target sequences, currently the focus of other epigenetic projects in our laboratory (see Supplementary Table S1 available online at www. BioTechniques.com and also at www. epigenomics.ca for details of the genes analyzed, primer sequences, and PCR conditions) were amplified by PCR. PCR products were electrophoresed on a $1 \%$ agarose gel and visualized using ethidium bromide staining. DNA fragments were excised and cleaned using the QIAquick ${ }^{\circledR}$ Gel Extraction kit (Qiagen). PCR products were subsequently $(i)$ directly sequenced using the reverse PCR primer with BigDye $^{\circledR}$ v3.1 sequencing chemistry (Applied Biosystems, Foster City, CA, USA); (ii) subjected to Ms-SNuPE analysis using the ABI PRISM ${ }^{\circledR}$ SNaPshot $^{\mathrm{TM}}$ approach (6); or (iii) cloned into the pGEM ${ }^{\circledR}$-T vector (Promega, Madison, WI, USA) and sequenced.

\section{RESULTS AND DISCUSSION}

This study sought to investigate whether WGA on bisulfite-treated DNA templates can be used to quantitatively assess DNA methylation levels and thus provide a possible solution to problems associated with low initial DNA amount and further degradation during and after sodium bisulfite treatment. WGA substantially increased the amount of usable post-bisulfite DNA. Using our PEP protocol after bisulfite treatment results in a large increase in usable template. We also tested a number of samples using an MDA-based WGA method (the REPLI-g kit), and this too proved to be a successful template for bisulfite-PCR amplification. 
A

WGA-

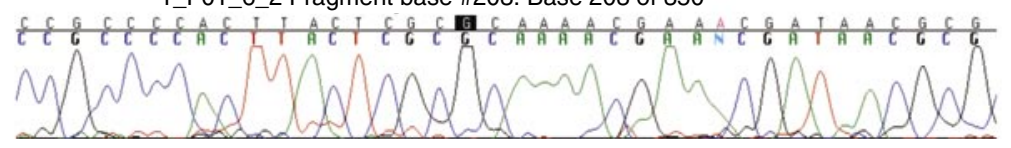

1 C01 3 3 Fragment base \#139. Base 139 of 291

WGA+

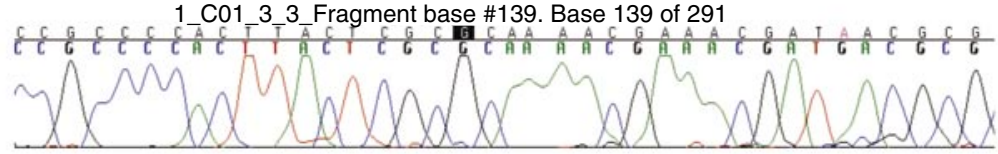

B

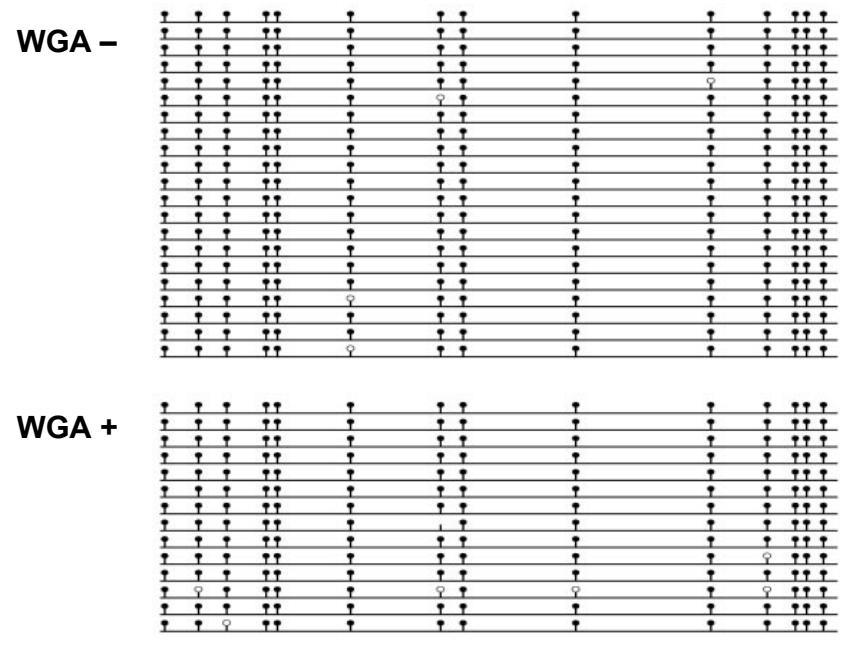

C

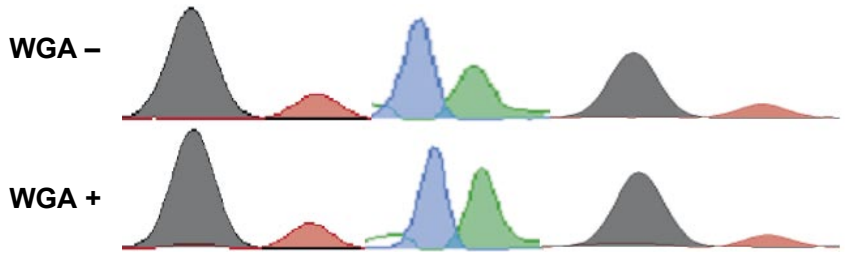

D

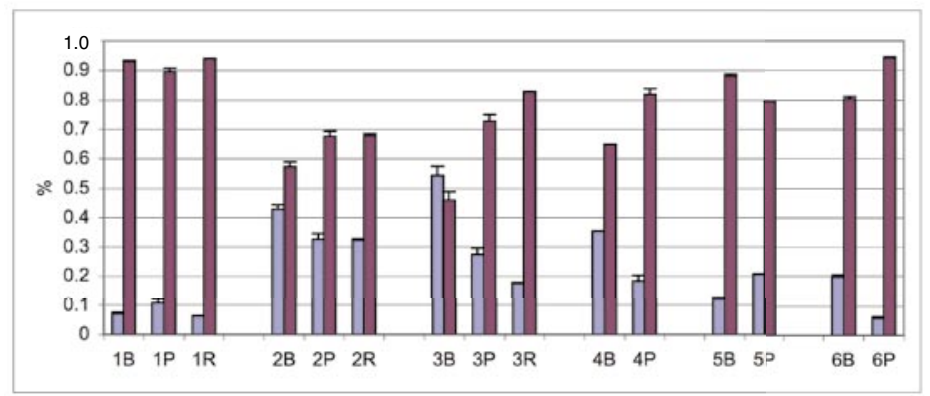

Figure 2. Examples of the three methylation profiling techniques used to compare data from non-whole genome amplification (WGA)/ WGA bisulfite DNA. (A) Direct sequencing chromatograms of INS PCR products generated from normal bisulfite-treated DNA and primerextension preamplification (PEP)-amplified bisulfite-treated DNA from the same individual. Sequencing was performed using the reverse PCR primer. (B) Sequencing of cloned PCR products from normal bisulfite-treated DNA (98\% methylated) and PEP-amplified bisulfite-treated DNA (97\% methylated) templates generated from the same individual. (C) Examples of SNaPshot traces for the $\mathrm{CDH} 13$ region interrogating three $\mathrm{CpG}$ sites on non-WGA bisulfite-treated DNA and PEP-amplified bisulfite-treated DNA from the same individual. Black (forward strand) and blue (reverse strand) peaks represent methylated cytosines, and red (forward strand) and green (reverse strand) peaks represent unmethylated cytosines. The degree of methylation is estimated by dividing the methylated peak height by the total peak height of both peaks. (D) Methylation estimates derived from $\mathrm{SNaPshot}$ traces for two $\mathrm{CpG}$ sites in $\mathrm{CDH} 13$ on templates generated from six DNA samples (numbered 1 through 6). Methylation estimates derived from WGA templates are similar to those produced by standard bisulfite-treated DNA templates. Blue bars denote the percentage of methylated cytosines, and red bars denote the percentage of unmethylated cytosines at this CpG site. B, non-WGA bisulfite-treated DNA; $\mathrm{P}$, PEP-amplified bisulfite-treated DNA; R, bisulfite-treated DNA amplified with the REPLI-g multiple displacement amplification (MDA) kit. 


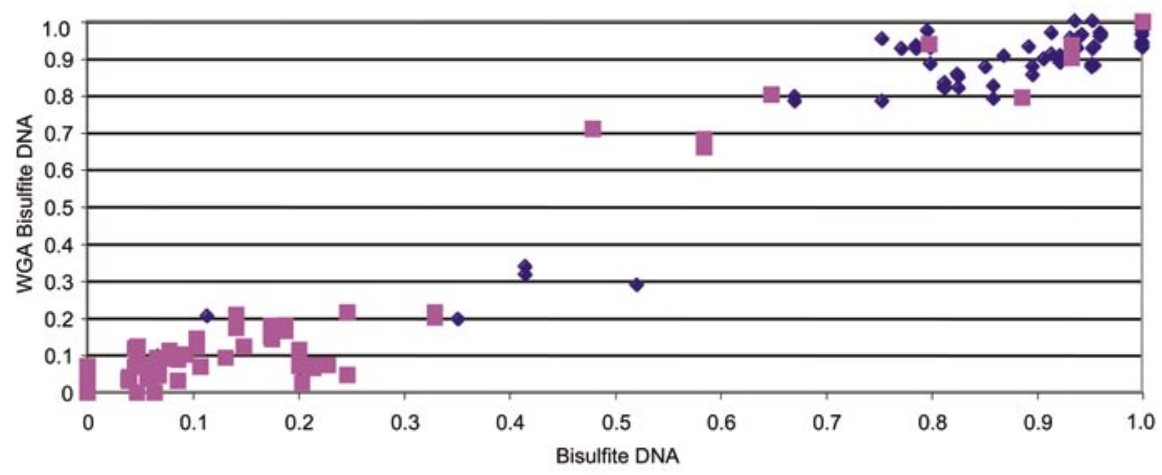

\% Methylated \% Unmethylated

Figure 3. Quantitative methylation data from all SNaPshot experiments on normal bisulfite-converted DNA versus whole genome amplification (WGA)-amplified bisulfite-treated DNA.

spectrophotometric analysis of 25 representative PEP-amplified products. The average yield from the pooled PEP replicates was $6.8 \mu \mathrm{g}(\mathrm{SD} 2.2 \mu \mathrm{g})$. We were able to obtain PCR products from standard sodium bisulfite-treated DNA and WGA-enriched templates generated using PEP and MDA. The overall locus-specific PCR success rate following WGA was no different to that observed using standard sodium bisulfite-treated DNA. In some instances, the intensity of the observed PCR bands was stronger from WGA templates compared with non-WGA bisulfite DNA. One potential problem with WGA methods is that even in the absence of input DNA, nonspecific amplification products may still be observed, resulting from amplification of the hexamers in the reaction. Furthermore, because WGA is highly efficient, contamination problems can be an issue. For this reason, we included a negative water control in each set of WGA reactions and used the products of these as a negative control for all subsequent PCR amplifications. For some products, agarose gel analysis of PCR performed on PEP templates revealed a faint background smear. We found that the post-WGA purification of PEP products using MinElute columns, to remove unused degenerate primers, makes downstream PCR amplifications somewhat cleaner, but did result in a slight reduction in available template due to the binding efficiency of the columns used.

In addition, the methylation profiles obtained from sodium bisulfite-treated
DNA amplified with both WGA methods were comparable to those obtained from non-WGA bisulfitetreated DNA, suggesting that for the specific genomic regions tested, WGA-DNA can be used to accurately estimate density of ${ }^{\mathrm{met}} \mathrm{C}$. Figure 2 shows examples from the three methylation profiling techniques performed on both sets of DNA templates. First, the direct sequencing of targeted bisulfite PCR products has been used as an important optimization of the bisulfite modification-based mapping of ${ }^{\text {met }} \mathrm{C}$, although the method is not very precise and does not provide clonespecific methylation profile (15). In our direct product sequencing experiments, WGA of bisulfite-treated DNA generated similar overall methylation patterns as in the non-WGA bisulfite DNA (Figure 2A), although four of the 10 products tested were not successfully directly sequenced using either type of template. In the PEP-amplified samples, direct sequencing data was improved when removal of excess degenerate primers was performed using MinElute columns.

Second, the sequencing of cloned bisulfite-treated DNA PCR products is often considered the gold standard in DNA methylation analysis. In this study, we analyzed products in the vicinity of four genes (ACATl, FLJ10458, GNL1, and ZNF498) in three individuals, sequencing 12-32 clones per individual for both nonWGA DNA and WGA-DNA. In the samples and products for which it was tested, the sequencing of cloned PCR products gave comparable methylation estimates from standard sodium bisulfite-treated DNA templates and PEP bisulfite DNA templates, with a $<5 \%{ }^{m e t} \mathrm{C}$ average difference between both sets of templates. An example comparison between non-WGA DNA and WGA-DNA cloned PCR products for one individual sample can be seen in Figure 2B.

Our third and most thorough investigation of WGA as a tool for quantitative methylation profiling was performed using Ms-SNuPE. The methylation status of 10 individual $\mathrm{CpG}$ sites was assessed in 6-10 individuals via MsSNuPE using the ABI PRISM SNaPshot method (6). Examples of SNaPshot traces for three $\mathrm{CpG}$ sites from a standard sodium bisulfite-treated DNA sample and those from the equivalent PEP-amplified sample can be seen in Figure 2C. MDA templates produced similarly accurate ${ }^{\mathrm{met}} \mathrm{C}$ estimates. Figure $2 \mathrm{D}$ shows the quantitative methylation profiles generated at two $\mathrm{CpG}$ sites across several samples using both sets of templates. It can be seen that while occasional differences in methylation estimates are observed (e.g., sample 3 in the bottom panel of Figure 2D), the general profiles obtained from standard sodium bisulfite-treated DNA templates and WGA bisulfite DNA templates are remarkably similar. Figure 3 highlights the quantitative methylation estimates from $\mathrm{SNaPshot} \mathrm{experiments} \mathrm{on} \mathrm{normal}$ bisulfite-converted DNA versus WGAamplified bisulfite-treated DNA across all $10 \mathrm{CpG}$ sites tested in this analysis. For each sample and for each $\mathrm{CpG}$ site tested, we calculated the difference in ${ }^{\mathrm{met}} \mathrm{C}$ percentage estimated from non-WGA and WGA bisulfite-treated DNA templates. The mean difference in methylation calculated from the two sets of template was $4 \%$, with a standard deviation of $5 \%$. The Pearson correlation between the two data sets was 0.979 .

Because a more homogeneous population of post-bisulfite DNA molecules is likely to result in less PCR bias, it would be expected that WGA accuracy is highest for totally methylated and totally unmethylated samples. To test whether WGA bisulfite-treated DNA gives accurate methylation estimates via Ms-SNuPE 
across the entire distribution of potential methylation levels, we repeated the analysis, first excluding samples showing $>95 \%$ methylation and $<5 \%$ methylation and, second, excluding samples showing $>80 \%$ methylation and $<20 \%$ methylation. In both cases, the correlation between metC estimates from non-WGA/WGA templates was high (95\% and $97 \%$, respectively), with the mean average difference between the two still within the limits expected from experiments using the SNaPshot approach (6) $(6 \% \pm 6 \%$ and $13 \% \pm 5 \%$, respectively). Finally, we examined the replicability of methylation data obtained from WGA bisulfite DNA templates. Experiments on four $\mathrm{CpG}$ sites in nine individual samples were repeated three times using the same WGA template. We observed a strong correlation between the data sets, with an average standard deviation in estimates of ${ }^{m e t} \mathrm{C}$ of $4.1 \%$. Overall, these data suggest that sodium bisulfitetreated DNA amplified by WGA could potentially be used as a template for the accurate quantitative methylation profiling of specific $\mathrm{CpG}$ sites.

To conclude, we have shown that WGA could be a useful method to overcome the problem of low initial amount of DNA and/or severe DNA degradation during conventional sodium bisulfite treatment in studies investigating DNA methylation. WGA is a relatively inexpensive process that can be optimized for high-throughput application and should enable the thorough investigation of methylation at numerous genomic locations on samples for which DNA availability is low. Bisulfite-treated DNA amplified using WGA can be used for a range of downstream quantitative methylation profiling techniques. While it should be acknowledged that WGA could potentially introduce biases into quantitative estimates of $\mathrm{CpG}$ methylation, our investigation suggests that such biases may not be a major problem. In this study we only tested a small number of loci, and it is thus difficult to judge how widely this method can be used to profile ${ }^{\mathrm{met}} \mathrm{C}$ across remaining regions of the genome. We propose that other laboratories should examine their target regions using WGA on bisulfitetreated DNA templates and compare their findings with non-WGA DNA. If there is additional evidence to suggest that the results obtained from WGA of bisulfite-treated DNA are consistent, this approach may become a routine step in the bisulfite modification-based mapping of methylated cytosines.

\section{ACKNOWLEDGMENTS}

This research has been supported by the Ontario Mental Health Foundation, Canadian Institutes for Health and Research (CIHR), National Institute of Mental Health (RO1 MH07412701), as well as the National Alliance for Research on Schizophrenia and Depression (NARSAD), and the Stanley Foundation. J.M. is funded by a CIHR postdoctoral fellowship.

\section{COMPETING INTERESTS STATEMENT}

The authors declare no competing interests.

\section{REFERENCES}

1.Henikoff, S. and M.A. Matzke. 1997. Exploring and explaining epigenetic effects. Trends Genet. 13:293-295.

2.El Maarri, O. 2003. Methods: DNA methylation. Adv. Exp. Med. Biol. 544:197-204.

3. Frommer, M., L.E. McDonald, D.S. Millar, C.M. Collis, F. Watt, G.W. Grigg, P.L. Molloy, and C.L. Paul. 1992. A genomic sequencing protocol that yields a positive display of 5-methylcytosine residues in individual DNA strands. Proc. Natl. Acad. Sci. USA 89:1827-1831.

4.Paul, C.L. and S.J. Clark. 1996. Cytosine methylation: quantitation by automated genomic sequencing and GENESCAN analysis. BioTechniques 21:126-133.

5. Gonzalgo, M.L. and P.A. Jones. 2002. Quantitative methylation analysis using methylation-sensitive single-nucleotide primer extension (Ms-SNuPE). Methods 27:128-133.

6. Kaminsky, Z.A., A. Assadzadeh, J. Flanagan, and A. Petronis. 2005. Single nucleotide extension technology for quantitative site-specific evaluation of metC/C in GC-rich regions. Nucleic Acids Res. 33:e95.

7. Grunau, C., S.J. Clark, and A. Rosenthal. 2001. Bisulfite genomic sequencing: systematic investigation of critical experimental parameters. Nucleic Acids Res. 29:e65.

8. Olek, A., J. Oswald, and J. Walter. 1996. A modified and improved method for bisulphite based cytosine methylation analysis. Nucleic Acids Res. 24:5064-5066.
9. Ballantyne, K.N., R.A. van Oorschot, and R.J. Mitchell. 2006. Comparison of two whole genome amplification methods for STR genotyping of LCN and degraded DNA samples. Forensic Sci Int. 2006 May 8; [Epub ahead of print].

10. Zhang, L., X. Cui, K. Schmitt, R. Hubert, W. Navidi, and N. Arnheim. 1992. Whole genome amplification from a single cell: implications for genetic analysis. Proc. Natl. Acad. Sci. USA 89:5847-5851.

11. Dean, F.B., S. Hosono, L. Fang, X. Wu, A.F. Faruqi, P. Bray-Ward, Z. Sun, Q. Zong, et al. 2002. Comprehensive human genome amplification using multiple displacement amplification. Proc. Natl. Acad. Sci. USA 99:5261-5266.

12. Kuivaniemi, H., S. Yoon, H. Shibamura, M. Skunca, S. Vongpunsawad, and G. Tromp. 2002. Primer-extension preamplified DNA is a reliable template for genotyping. Clin. Chem. 48:1601-1604.

13. Hosono, S., A.F. Faruqi, F.B. Dean, Y. Du, Z. Sun, X. Wu, J. Du, S.F. Kingsmore, et al. 2003. Unbiased whole-genome amplification directly from clinical samples. Genome Res. 13:954-964.

14. Clark, S.J., J. Harrison, C.L. Paul, and M. Frommer. 1994. High sensitivity mapping of methylated cytosines. Nucleic Acids Res. 22:2990-2997.

15. Rakyan, V.K., T. Hildmann, K.L. Novik, J. Lewin, J. Tost, A.V. Cox, T.D. Andrews, K.L. Howe, et al. 2004. DNA methylation profiling of the human major histocompatibility complex: a pilot study for the human epigenome project. PLoS Biol. 2:e405.

Received 20 June 2006; accepted 4 August 2006.

Address correspondence to Arturas Petronis, Centre for Addiction and Mental Health, 250 College Street, Toronto, ON, M5T 1R8, Canada. e-mail: arturas_petronis@camh.net

To purchase reprints of this article, contact: Reprints@BioTechniques.com 